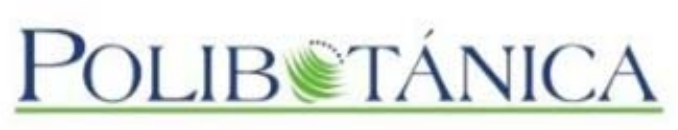

\title{
Polibotánica
}

ISSN electrónico: 2395-9525

polibotanica@gmail.com

Instituto Politécnico Nacional

México

http:www.polibotanica.mx

\section{LISTA ACTUALIZADA DE LAS BIGNONIACEAE DE TABASCO, MÉXICO}

\section{AN UPDATED CHECKLIST OF THE BIGNONIACEAE FROM TABASCO, MEXICO}

\section{Burelo-Ramos, C.M. y M.A. Guadarrama-Olivera.} LISTA ACTUALIZADA DE LAS BIGNONIACEAE DE TABASCO, MÉXICO. AN UPDATED CHECKLIST OF THE BIGNONIACEAE FROM TABASCO, MEXICO.

\section{POLIBETANICA}

Instituto Politécnico Nacional
Núm. 48: 1-12 México. Julio 2019

DOI: 10.18387 polibotanica.48.1 
LISTA ACTUALIZADA DE LAS BIGNONIACEAE DE TABASCO, MÉXICO

\title{
AN UPDATED CHECKLIST OF THE BIGNONIACEAE FROM TABASCO, MEXICO
}

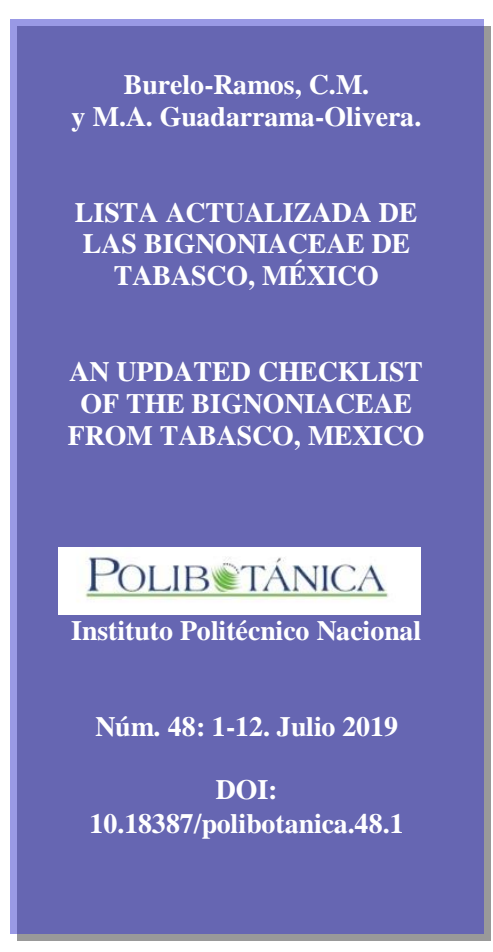

\author{
C.M. Burelo-Ramos / carlos.burelo@ujat.mx \\ M.A. Guadarrama-Olivera \\ Herbario UJAT, División Académica de Ciencias Biológicas. \\ Universidad Juárez Autónoma de Tabasco. \\ Carretera Villahermosa-Cárdenas km 1.5, Villahermosa Tabasco, México.
}

RESUMEN: El presente trabajo enlista las especies de Bignoniaceae distribuidas en el estado de Tabasco, utilizando la nomenclatura propuesta bajo los nuevos esquemas clasificatorios generados por las filogenias reconstruidas recientemente. La recolecta en todo el estado de Tabasco, la revisión de los Herbarios estatales y nacionales, la revisión de ejemplares de herbarios en línea procedentes de Tabasco fue la base para este listado, las especies fueron corroboradas y determinadas utilizando trabajos de Bignoniaceae disponibles (Veracruz y Guatemala) y la consulta de la revisión de la "Allianza Tabebuia" y la sinopsis de la tribu Bignonieae, además de consultar "World Checklist of Bignoniaceae".

Bignoniaceae está representada por 47 especies y 24 géneros, de las cuales cuatro especies son nuevos registros para el Estado. Los géneros mas diversos son Bignonia L, Amphitecna Miers y Fridericia Mart. con cinco especies cada una. Las lianas son las más abundantes con 30 especies, seguida de los árboles con 14 especies y los arbustos con tres. No existen especies de distribución restringida a Tabasco. Handroanthus chrysanthus S.O. Grose se enlista en la Norma oficial mexicana bajo la categoría de Amenazada. En Tabasco se resguarda el 80\% de los géneros y el $61 \%$ de las especies de Bignoniaceae de México.

Palabras clave: biodiversidad, inventario botánico, flora de México, sistemática, taxonomía.

\begin{abstract}
This paper lists the Biognaceae species distributed in Tabasco state, using the proposed nomenclature based on the new classification schemes generated by recently reconstructed phylogenies. In order to complete the list, collects across all Tabasco state were made, hebarium of the state and the country were reviewed, the review on line of specimens of the herbarium coming from Tabasco was the base for this list, the species were determined and corroborated using available works of Bignoniaceae (Veracruz and Guatemala) and the consultation of the "Allianza Tabebuia" and the synopsis of the Bignonieae tribe, besides the "World Checklist of Bignoniacead" was consulted. Bignoniaceae is represented by 47 species and 24 genus, four of those species are new records for the state. The more diverse genus are Bignonia L. Amphitecna Miers y Fredericia Mart. with five species each. The lianas are the most abundant with 30 species, followed by the trees with 14 species and the shurbs with 3. There are no species of restricted distribution in Tabasco. Handroanthus chrysanthus S.O. Grose is listed in the Official Mexican Norm under the threatened category. The Bignoniaceae record in Tabasco represents that the state protects $80 \%$ of the genera and $61 \%$ of the species of Bignoniaceae of Mexico.
\end{abstract}

Key words: biodiversity, botanical inventory, Mexican flora, systematics, taxonomy.

\section{INTRODUCCIÓN}


La familia Bignoniaceae reúne cerca de 82 géneros y 827 especies (Lohmann \& Ulloa, 2016) de árboles, algunos arbustos y principalmente lianas. Las lianas Bignoniaceae son extremamente abundantes y diversas en el Neotrópico y la familia es considerada una de las familias de lianas más importantes en Centroamérica (Gentry, 1973). La familia es monofilética incluye ocho clados principales: las tribus Bignonieae, Catalpeae, Jacarandeae, Oroxyleae, Tecomeae y Tourrettieae, y los clados informales "Allianza Tabebuia" y "Clado Neotropical" (Olmstead, Zjhra, \& Lohmann, 2009).

Varios trabajos reconstruyeron las relaciones filogenéticas al interior de las tribus de Bignoniaceae, especialmente Coleeae (Zjhra, Sytsma, \& Olmstead, 2004), Bignonieae (Lohmann, 2006) y el clado "Tabebuia Alliance" (Grose \& Olmstead, 2007b). Estos trabajos han sido utilizados como base para establecer nuevas clasificaciones genéricas en Bignonieae (Lohmann \& Taylor, 2015) y el clado "Allianza Tabebuia" (Grose \& Olmstead, 2007a). Aparte de los estudios filogenéticos al nivel tribal, otros estudios han establecido las relaciones interespecificas en géneros de varias tribus como Incarvillea (Oroxyleae) (Chen, Guan, Olmstead, \& Cronk, 2005), Catalpa (Catalpeae) (Li, 2008), Lundia (Bignonieae) (Kaehler, Michelangeli, \& Lohmann, 2012), Tynanthus (Bignonieae) (Medeiros \& Lohmann, 2015) у Dolichandra (Bignonieae) (Fonseca \& Lohman, 2015).

Las Bignoniaceae han sido tratadas en varias floras y listas de especies para Latino América. Por ejemplo, Panamá (Gentry, 1973), Guatemala (C. Standley \& O., 1977), Ecuador (Gentry, 1977), Guyanas (Gentry, 1978), Venezuela (Gentry, 1983; Lohmann, 2008), Cono Sur (Arbo \& Lohmann, 2008), Colombia (Gentry, 2009), Brasil (Lohmann, 2010) y Bolivia (Lohmann \& Fuentes, 2015). Además, una lista de las Bignoniaceae para el Mundo también ya está disponible en línea (Lohmann \& Ulloa, 2016), en esta obra, 30 géneros y 77 especies de Bignoniaceae son listadas para México, aunque Villaseñor (2016) menciona la presencia de 26 géneros y 68 especies. Mientras que Ibarra et al. (2015) menciona para México la presencia de 19 géneros y 39 especies solo de lianas (Tribu Bignoniea). Aparte de esto, obras taxonómicas detalladas de las Bignoniaceae están disponibles para los estados de Veracruz, donde se encuentran 45 especies (Gentry, 1982), Oaxaca donde se distribuyen 59 especies (GarcíaMendoza \& Meave, 2012), Chiapas donde se enlistan 58 especies (Breedlove, 1986), y la Península de Yucatán donde se han documentado 31 (Fernández-Concha, Tapia-Muñoz, Duno De Stefano, \& Ramírez, 2010).

Para el estado de Tabasco existen cuatro listas que están disponibles, una de las cuales presenta 15 géneros y 24 especies (Cowan, 1983), otra enlista 19 géneros y 35 especies (Pérez, Sousa, Hanan, Chiang, \& Tenorio, 2005). Mientras que del trabajo de Villaseñor (2016) podemos reconocer la presencia de 21 géneros y 41especies. Ibarra et al. (2015) menciona que para Tabasco se distribuyen 13 géneros y 19 especies exclusivamente de lianas, Además de esto, Gentry (1980) reporta tres géneros y seis especies de Crescentieae (= "Allianza Tabebuia" sensu Olmstead et al., 2009) nativas de Tabasco, en su revisión de la tribu; cinco géneros y cinco especies de Tecomeae (= Allianza Tabebuia" sensu Olmstead et al., 2009) en su revisión de este grupo (Gentry, 1992). Además, (Lohmann \& Taylor, 2015) listan 11 géneros y 23 especies de Bignonieae nativas de Tabasco en su sinopsis de la tribu Bignonieae. Recientemente se registró en Tabasco, la presencia de Amphitecna donell-smithii (Sprague) L.O. Williams, (Burelo-Ramos \& Guadarrama-Olivera, 2016), que se consideraba endémica a la zona del Petén en Guatemala, lo que representa un nuevo registro para la flora de México. Así mismo se registró la presencia de Mansoa sagotii (Bureau \& K. Schum.) M.M. SilvaCastro, para Tabasco (Silva-Castro \& Queiroz, 2016).

En el presente estudio se presenta una revisión actualizada de las Bignoniaceae como una contribución al proyecto Flora de Tabasco, producida por el Herbario Universidad Juárez Autónoma de Tabasco (UJAT). 


\section{MÉTODOS}

Este estudio basado en recolecciones intensivas realizadas en diversas áreas de Tabasco, la consulta de la literatura especializada, y la revisión de ejemplares de los herbarios CSAT, MEXU, UJAT y XAL (abreviaciones según Thiers, 2016, actualizado continuamente). Este trabajo es la conclusión del estudio iniciado entre 1998 y 2000 con recolectas de campo intensivas es toda la zona de estudio dirigidas a la familia Bignoniaceae y una segunda etapa, de 2010 a 2013, en la cual se estudiaron las plantas trepadoras de Tabasco. También, se incluyen las colectas entre estos periodos, productos de otros proyectos que desarrolla el Herbario UJAT en el estado. El material colectado fue depositado en la colección del herbario UJAT y sus duplicados fueron intercambiados con herbarios nacionales. Para la corroboración y la determinación de este material se utilizaron las publicaciones de Bignoniaceae de Veracruz (Gentry, 1982) y Flora de Guatemala (C. Standley \& O., 1977). El listado fue estructurado de acuerdo a la propuesta de el "World Checklist of Bignoniaceae" (Lohmann \& Ulloa, 2016), las revisiones disponibles para la "Allianza Tabebuia" (Gentry, 1980, 1982) y la sinopsis de la tribu Bignonieae (Lohmann \& Taylor, 2015).

\section{Resultados}

En Tabasco, Bignoniaceae está representada por 20 géneros y 43 especies nativas, así como por cuatro géneros y especies introducidas cultivadas como ornamentales, lo que suma un total de 47 especies y 24 géneros. De estas especies cuatro especies son nuevos registros para el Estado (tabla 1). Los géneros más diversos son Bignonia L, Amphitecna Miers y Fridericia Mart. con cinco especies cada uno, Adenocalymma Mart. ex Meisn. y Dolichandra Cham. con tres especies cada uno (gráfica 1). En lo que respecta al hábito, se encontraron 14 árboles, tres arbustos y 30 trepadoras (lianas) (tabla 1).

La mayoría de las especies encontradas presentan amplia distribución o se encuentran compartidas con los estados de Veracruz, Chiapas y con Guatemala. No se hallaron especies endémicas para Tabasco. Se destaca la presencia de Handroanthus chrysanthus S.O. Grose que se enlista en la Norma oficial mexicana NOM-059-SEMARNAT-2010 (como Tabebuia chrysantha (Jacq.) G. Nicholson) bajo la categoría de Amenazada.

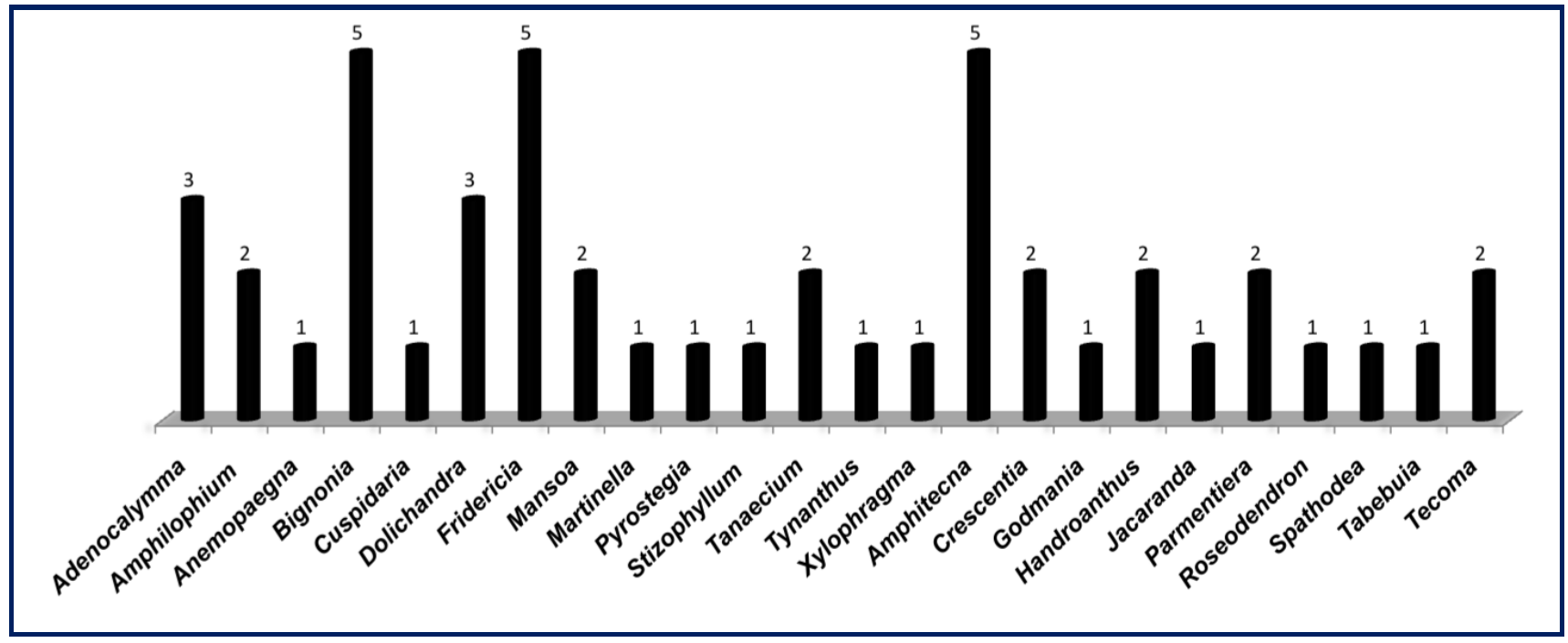

Grafica 1. Número de especies por géneros de la familia Bignoniaceae en Tabasco, México. 


\section{DISCUSIÓN}

El reporte de cuatro nuevos registros incrementa en $8.5 \%$ el total de las especies de Bignoniceae anteriormente reportadas para Tabasco. Bignoniaceae ha sido considerada como una de las familias más importantes de lianas de Centroamérica (Gentry, 1983). En Tabasco ocupa el cuarto lugar entre las familias de plantas trepadoras (Burelo, et al. en preparación). Gentry (1991) y Acevedo-Rodríguez (2005) señalan que existe un vacío en el conocimiento de las plantas trepadoras en general y que han recibido relativamente poca atención, siendo poco recolectadas y por lo tanto pobremente representadas en los herbarios nacionales e internacionales. Las especies de árboles y arbustos de Bignoniaceae representan el 35.5\% de las Bignoniaceae de Tabasco y a pesar de que los árboles son las formas de vida más conspicua y tomados en cuenta en estudios primarios y en los estudios florísticos, las colectas hechas lograron un incremento en dos especies.

Handroanthus chrysanthus (Jacq.) S.O. Grose se encuentra categorizada como Amenazada de acuerdo a la NOM-059-SEMARNAT-2010 (como Tabebuia chrysantha (Jacq.) G. Nicholson). Esta especie se conoce únicamente de un espécimen recolectado en 1972 y hasta a la fecha no se ha vuelto a recolectar en el estado, por lo que su situación puede considerar como crítica o posiblemente extinta, esto dado a la perdida de las selvas que se ha presentado en el estado, aunque es común en Chiapas, Veracruz y existen reportes en la Península de Yucatán.

\section{CONCLUSIONES}

La presente contribución representa un primer paso para el estudio de esta familia para la flora de México. A pesar de 35 años de exploración botánica en la zona de estudio y encontrar especies que no habían sido reportadas (cuatro en este caso) indica que aún queda trabajo campo y sistemático por realizar.

En México se distribuyen 30 géneros y 77 especies de Bignoniaceae, tomando como referencia el "World Checklist de las Bignoniaceae" (Lohmann \& Ulloa, 2016), el cual es único listado completo y nomenclaturalmente soportado por hipótesis filogenéticas. Si tomamos esta riqueza de géneros y especies, concluimos que en el estado de Tabasco se encuentran representados el $80 \%$ de los géneros y el $61 \%$ de las especies de Bignoniaceae de México. Sin embargo, el aumento de $8.5 \%$ de las especies conocidas para Tabasco, hace necesario reconsiderar la riqueza conocida de esta familia en México, ya que, con más revisiones a herbarios locales y regionales, trabajo de campo, además del trabajo pendiente de actualizar la nomenclatura de esta importante familia, puede aumentar el número de especies de Bignoniaceae en México.

\section{AgRadecimientos}

A Neil Ebeth Meled Morales Rodríguez por su apoyo en la edición de la bibliografía. A la Universidad Juárez Autónoma de Tabasco por su apoyo en el desarrollo del proyecto. Publicación financiada con recursos PFCE 2019.

\section{LITERATURA CITADA}

Acevedo-Rodríguez, P. (2005). Vines and climbing plants of Puerto Rico and the Virgin Islands. Contributions from the U.S. National Herbarium (Vol. 51). Washington DC. Retrieved from http://www.nmnh.si.edu/botany/pubs/CUSNH/vol51web.pdf

Arbo, M. M., \& Lohmann, L. G. (2008). Bignoniaceae. In F. O. Zuloaga, O. Morrone, \& M. J. Belgrano (Eds.), Catálogo de las Plantas Vasculares del Cono Sur (Vol. 2, pp. 1581- 
1627). Missouri Botanical Garden Press, EUA.

Breedlove, D. E. (1986). Listados florísticos de México. IV Flora de Chiapas. Retrieved from http://www.ibiologia.unam.mx/BIBLIO68/fulltext/lfl4.html

Burelo-Ramos, C. M., \& Guadarrama-Olivera, M. de los Á. (2016). Primer registro de Amphitecna donell smithii (Bignoniaceae) y calve de identificación del género para México. Ecosistemas y Recursos Agropecuarios, 3 (8), 225-228.

Chen, S. K., Guan, Z. Z., Olmstead, R. G., \& Cronk, Q. (2005). Molecular phylogeny of Incarvillea (Bignoniaceae) based on ITS and trnL-F sequences. American Journal of Botany, 92, 625-633.

Cowan, P. C. (1983). Listado florísticos de México. I. Flora de Tabasco. Retrieved from http://www.ibiologia.unam.mx/BIBLIO68/fulltext/lfl1.html

Fernández-Concha, G. C., Tapia-Muñoz, J. L., Duno De Stefano, R., \& Ramírez, I. M. (2010). Flora ilustada de la Península de Yucatán: Listado Florístico. Mérida. Yucatán: Centro de Investigación Científica de Yucatán, A. C.

Fonseca, L. H. M. F., \& Lohman. (2015). Biogeography and evolution of Dolichandra (Bignonieae, Bignoniaceae). Botanical Journal of the Linnean Society, 179, 403-420.

García-Mendoza, A. J., \& Meave, J. A. (2012). Diversidad Florística de Oaxaca: de musgos a angiospermas (Colecciones y lista de especies). México: Universidad Nacional Autónoma de México - Comisión Nacional para el Conocimiento de la Biodiversidad.

Gentry, A. H. (1973). Bignoniaceae. Flora of Panamá. Annals of the Missouri Botanical Garden Gard., 60, 781-997.

Gentry, A. H. (1977). Bignoniaceae. In P. Martinet, P. Harling, \& B. Sparre (Eds.), Flora de Ecuador (7th ed., p. 172). Stockholm: University of Goteberg and Riksmuseum.

Gentry, A. H. (1978). Bignoniaceae. Botany of the Guayana Highland. Memoirs New York. Botanical Garden, 29, 245-283.

Gentry, A. H. (1980). Bignoniaceae Part I (Crescentieae and Tourrettieae). Flora Neotrópica, $25(1), 1-131$.

Gentry, A. H. (1982). Bignoniaceae. Flora de Veracruz, 24, 1-122.

Gentry, A. H. (1983). Bignoniaceae. Flora de Venezuela, 8 (4), 7-433.

Gentry, A. H. (1991). The distribution and evolution of climbing plants. In F. E. Putz \& H. A. Mooney (Eds.), The biology of vines (pp. 3-49). Cambridge, Australia.: Cambridge University Press.

Gentry, A. H. (1992). Bignoniaceae Part II (Tecomae). Flora Neotrópica, 25 (2), 1-371.

Gentry, A. H. (2009). Bignoniaceae. In E. Forero, J. Betancur, G. Galeno, \& J. Aguirre (Eds.), Flora de Colombia (25th ed., p. 462). Colombia: Instituto de Ciencias Naturales. Universidad Nacional de Colombia.

Grose, S., \& Olmstead, R. G. (2007a). Taxonomic revisions in the polyphyletic genus Tabebuia s.1. (Bignoniaceae). Systematic Botany, 32, 660-670.

Grose, S. O., \& Olmstead, R. G. (2007b). Evolution of a charismatic neotropical clade: Molecular phylogeny of Tabebuia s. 1., Crescentieae, and allied genera (Bignoniaceae). Systematic Botany, 32, 650-659.

Ibarra-Manríquez, G., Rendón-Sandoval, F., Cornejo-Tenorio, G., \& Carrillo-Reyes, P. (2015). Lianas of México. Botanical Sciences, 93(3), 365-417.

Kaehler, M. F., Michelangeli, F., \& Lohmann, L. G. (2012). Phylogeny of Lundia based on molecular and morphological characters. Taxon, 61, 368-380.

Li, J. (2008). Phylogeny of Catalpa (Bignoniaceae) inferred from sequences of chloroplast ndhF and nuclear ribosomal DNA. Journal of Systematic and Evolution, 46, 341-348.

Lohmann, L. G. (2006). Untangling the phylogeny of neotropical lianas (Bignonieae, Bignoniaceae). American Journal of Botany, 93, 304-318.

Lohmann, L. G. (2008). Bignoniaceae. In O. Hocke, P. E. Berry, \& O. Huber (Eds.), Nuevo Catálogo de la Flora de Venezuela (pp. 270-278). Caracas, Venezuela: Fundación Instituto Botánico de Venezuela Dr. Tobias Lasser.

Lohmann, L. G. (2010). Bignoniaceae. In R. C. Forzza, J. F. A. Baumgartz, C. E. M. Bicudo, A. A. Carvalho Jr, A. Costa, C. D. P., D. Zappi (Eds.), Catálogo de Plantas e Fungos do Brasil (pp. 758-772). Rio de Janeiro, Brasil: Jardín Botánico de Rio de Janeiro. 
Recibido:

6/febrero/2018

Aceptado:

6/mayo/2019
Lohmann, L. G., \& Fuentes, A. (2015). Bignoniaceae. In P. Jorgensen, M. H. Nee, \& S. G. Beck (Eds.), Catálogo de las plantas vasculares de Bolivia (pp. 388-403). Sant Louis, EUA: Missouri Botanical Garden Press.

Lohmann, L. G., \& Taylor, C. M. (2015). A new generic classification of tribe Bignonieae (Bignoniaceae). Annals of the Missouri Botanical Garden Gard.2, 99, 348-489.

Lohmann, L. G., \& Ulloa, C. U. (2016). iPlants prototype checklist. Retrieved from http://www.iplants.org/

Medeiros, M. C. P., \& Lohmann, L. G. (2015). Phylogeny and biogeography of Tynanthus (Bignonieae, Bignoniaceae). Molecular Phylogenetics and Evolution, 85, 32-40.

Olmstead, R. G., Zjhra, M. L., \& Lohmann, L. G. (2009). Molecular phylogeny and classification of Bignoniaceea. American Journal of Botany2, 96, 1731-1743.

Pérez, L. A., Sousa, M., Hanan, A. M., Chiang, F., \& Tenorio, P. (2005). Vegetación terrestre. In J. Bueno, F. Álvarez, \& S. Santiago (Eds.), Biodiversidad del estado de Tabasco (Instituto, pp. 65-110). D.F. México.

SEGOB. (2010). NOM-059-SEMARNAT-2010, Protección ambiental-Especies nativas de México de flora y fauna silvestres-Categorías de riesgo y especificaciones para su inclusión, exclusión o cambio-Lista de especies en riesgo. Retrieved from http://dof.gob.mx/nota_detalle.php?codigo $=5173091 \&$ fecha $=30 / 12 / 2010$

Silva-Castro, M. M., \& Queiroz, L. P. (2016). Five new species of Mansoa DC. (Bignoniaceae) from South America. Phytotaxa, 258, 49-62.

Standley, C., \& O., W. L. (1977). Bignoniaceae. In P. C. Standley, L. O. Williams, \& D. N. Gibson (Eds.), Flora de Guatemala (24th ed., pp. 153-321). Fieldiana Botany.

Thiers, B. (n.d.). Index Herbariorum: A global directory of public herbaria and associated staff. Retrieved February 1, 2016, from http://sweetgum.nybg.org/ig/

Villaseñor, J. L. (2016). Catálogo de las plantas vasculares nativas de México. Revista Mexicana de Biodiversidad, 87, 559-902

Zjhra, M. L., Sytsma, K. J., \& Olmstead, R. G. (2004). Delimitation of Malagasy tribe Coleeae and implications for fruit evolution in Bignoniaceae inferred from a chloroplast DNA phylogeny. Phytotaxa, 245, 55-67. 
Tabla 1. Lista actualizada de especies de Bignoniaceae de Tabasco. Las especies presentadas en negrito representan nuevos registros para el estado; las demás especies de la "Allianza Tabebuia” y tribu Bignonieae han sido listadas para el estado por Cowan (1980), Pérez et al. (2005),

Gentry (1980, 1992), Ibarra et al. (2015). * indica especies introducidas cultivadas. L: lianas, A: árbol, Ar: arbusto. FV: forma de vida.

\begin{tabular}{|c|c|c|c|}
\hline Especies & $\begin{array}{c}\text { Taxa incluidas en otros listados como } \\
\text { sinónimos o nombre en uso de } \\
\text { material de Tabasco } \\
\end{array}$ & Ejemplares examinados & $\mathbf{F V}$ \\
\hline $\begin{array}{l}\text { Adenicalymma apurense (Kunth) } \\
\text { Sandwith }\end{array}$ & & Cantú R. 148 (MEXU). & $\mathbf{L}$ \\
\hline A. inundatum Mart. ex DC. & $\begin{array}{l}\text { Ibarra } \text { et al. (2015), Villaseñor (2016): } \\
\text { A. inundatum }\end{array}$ & $\begin{array}{l}\text { C. M. Burelo R. } 40 \text { (UJAT); F. Ventura A. (CSAT); G. Ortiz G. \& TCI-1, } \\
5098 \text { (UJAT); G. Ortiz G. y TCI-1 } 5045 \text { (UJAT); M.Á. Guadarrama O., } \\
\text { G.V. Ruiz L. y C.M. Burelo R. } 6770 \text { (UJAT). }\end{array}$ & $\mathbf{L}$ \\
\hline A. sousae A.H. Gentry & $\begin{array}{l}\text { Ibarra } \text { et al. (2015), Villaseñor (2016): } \\
\text { A. sousae }\end{array}$ & M.Á. Guadarrama 373 (UJAT, MO). & \\
\hline $\begin{array}{l}\text { Amphilophium crucigerum (L.) L.G. } \\
\text { Lohmann }\end{array}$ & $\begin{array}{l}\text { Cowan (1983): Pithecoctenium } \\
\text { echinatum (Jacq.) Schum. } \\
\text { Pérez et al., (2005): P. crucigerum (L.) } \\
\text { A.H. Gentry, P. echinatum } \\
\text { Ibarra et al. (2015), Villaseñor (2016): } \\
\text { Amphilophium crucigerum }\end{array}$ & $\begin{array}{l}\text { A. Sol S. } 166 \text { (UJAT); F. Ventura L. } 20937 \text { (MEXU); G. Ortiz G. } 5302 \\
\text { (UJAT); G. Ortiz G. et al. } 5299 \text { (UJAT); A.M. Hanan A. } 766 \text { (MEXU, } \\
\text { UJAT). }\end{array}$ & $\mathbf{L}$ \\
\hline A. paniculatum (L.) Kunth & $\begin{array}{l}\text { Ibarra } \text { et al. }(\mathbf{2 0 1 5}), \text { Villaseñor (2016): } \\
\text { A. paniculatum }\end{array}$ & $\begin{array}{l}\text { Kunth M.Á. Guadarrama O. \& G. Ortiz G. } 5433 \text { (UJAT); M.Á. } \\
\text { Guadarrama O. \& G. Ortiz G. } 5428 \text { (CSAT). } \\
\end{array}$ & $\mathbf{L}$ \\
\hline Amphitecna apiculata A.H. Gentry & $\begin{array}{l}\text { Cowan (1983), Pérez et. al., (2005), } \\
\text { Villaseñor (2016): A. apiculata }\end{array}$ & $\begin{array}{l}\text { A. Novelo R. 196, (MEXU); G. Ortiz G. \& Gpo. TCI-I Chapingo } 2189 \\
\text { (UJAT); P. Alcudia G. 7, (UJAT); J. Jiménez s.n. (UJAT); M.A. Magaña } \\
\text { A. \& S. Zamudio R., 360, (UJAT); M. R. Niño C. \& C.P. Cowan } 2968 \\
\text { (CSAT, MEXU); E.S. López H., L. Lozada \& R. Gutiérrez, 154, (UJAT); } \\
\text { G. Ramos E. \& C. P. Cowan 2690 (MEXU, CSAT); A. Sol S. 838 } \\
\text { (UJAT); González L. \& L. Hernández P. } 130 \text { (MEXU). }\end{array}$ & $\mathbf{A}$ \\
\hline $\begin{array}{l}\text { A. donell-smitti (Sprague) L.O. } \\
\text { Williams }\end{array}$ & Villaseñor (2016): A. donell-smitti & C.M. Burelo R. et al.46 (MO, UJAT). & $\mathbf{A}$ \\
\hline A. latifolia (Mill.) A.H. Gentry & $\begin{array}{l}\text { Cowan (1983), Pérez et al. (2005): } \\
\text { Amphitecna obovata (Benth.) L.O. } \\
\text { Williams, Enallagma latifolia (Mill.) } \\
\text { Small } \\
\text { Villaseñor (2016): } \text { A. latifolia }\end{array}$ & O. Castillo A., 762 (MEXU, UJAT). & $\mathbf{A}$ \\
\hline $\begin{array}{l}\text { A. macrophylla (Seem.) Miers ex } \\
\text { Baill. }\end{array}$ & $\begin{array}{l}\text { Pérez et al., (2005), Villaseñor (2016): } \\
\text { A. macrophylla }\end{array}$ & $\begin{array}{l}\text { A.M. Hanan A., M.Á. Guadarrama O., G. Ortiz G., L. Jiménez \& H. } \\
\text { Cervantes, } 334 \text { (MEXU, UJAT); G. Ramos E. \& M.Á. Guadarrama O. } \\
662 \text { (UJAT). }\end{array}$ & $\mathrm{A}$ \\
\hline
\end{tabular}




\begin{tabular}{|c|c|c|c|}
\hline Especies & $\begin{array}{c}\text { Taxa incluidas en otros listados como } \\
\text { sinónimos o nombre en uso de } \\
\text { material de Tabasco }\end{array}$ & Ejemplares examinados & $\mathbf{F V}$ \\
\hline A. regalis (Linden) A.H. Gentry & Villaseñor (2016): A. regalis & $\begin{array}{l}\text { González L., León C. y V. Garza s/n., (MEXU); G. Ramos E. et al. } 734 \\
\text { (UJAT). }\end{array}$ & $\overline{\mathbf{A}}$ \\
\hline Anemopaegna chrysanthum Dugand & Villaseñor (2016): A. chrysanthum & $\begin{array}{l}\text { M.Á. Guadarrama O., G.V. Ruíz L. \& S. López C. (UJAT); M.Á. } \\
\text { Guadarrama O., G. Ortiz G. et al. } 6282 \text { (UJAT). }\end{array}$ & $\mathbf{L}$ \\
\hline Bignonia aequinoctialis $\mathrm{L}$. & $\begin{array}{l}\text { Cowan (1983), Pérez et al. (2005): } \\
\text { Cydista aequinoctialis }(\text { L.) Miers } \\
\text { Ibarra } \text { et al. } \text { (2015), Villaseñor (2016): } \\
\text { B. aequinoctialis }\end{array}$ & $\begin{array}{l}\text { Centeno R. 45, 7/X/1976 (CSAT); F. Ventura A. } 20154 \text { (CSAT); M.Á. } \\
\text { Guadarrama O. } 505 \text { (UJAT); J.N. Rovirosa A. 149, (MEXU); H. Puig } \\
585 \text { (CSAT); F. Ventura A. 20172 (CSAT); Fernández \& S. Zamudio R. } \\
1002 \text { (CSAT); H. Puig } 585 \text { (CSAT); F. Menéndez } 259 \text { (XAL); A. Novelo } \\
\text { R. } 125 \text { (XAL); E. Matuda } 3421 \text { (MICH). }\end{array}$ & $\mathbf{L}$ \\
\hline B. diversifolia Kunth & $\begin{array}{l}\text { Cowan (1983), Pérez et al. (2005): } \\
\text { Cydista diversifolia (Kunth) } \\
\text { Ibarra } \text { et al. } \text { (2015), Villaseñor (2016): } \\
\text { B. diversifolia }\end{array}$ & F. Ventura A. 20799 (CSAT). & $\mathbf{L}$ \\
\hline $\begin{array}{l}\text { Bignonia neoheterophylla L.G. } \\
\text { Lohmann }\end{array}$ & $\begin{array}{l}\text { Ibarra et al. (2015), Villaseñor (2016): } \\
\text { B. neoheterophylla }\end{array}$ & M. A. Magaña A., 1756 (UJAT); M. A. Magaña A. 1627. (UJAT). & \\
\hline Bignonia noterophila Mart. ex DC. & $\begin{array}{l}\text { Cowan (1983): Clytstoma ocositense } \\
\text { (Donn.Sm.) Seibert } \\
\text { Pérez et al. }(\mathbf{2 0 0 5 )} \text { : C. binatum (Thunb.) } \\
\text { Sandwith } \\
\text { C. ocositense } \\
\text { Ibarra et al. (2015), Villaseñor (2016): } \\
\text { Bignonia binata Thunb. }\end{array}$ & $\begin{array}{l}\text { MA Magaña A. y R. Curiel } 205 \text { (CSAT, UJAT); M.Á. Guadarrama O. y N. } \\
\text { Muñiz Ch. } 6558 \text { (UJAT); M.Á. Guadarrama O. et al. 6476 (UJAT); M.Á. } \\
\text { Guadarrama O. et al. } 6769 \text { (UJAT); M.Á. Guadarrama O. et al. 6467 } \\
\text { (UJAT); M.A. Magaña A. y M.Á. Guadarrama O. } 2271 \text { (UJAT); M.Á. } \\
\text { Guadarrama O., G. Ortiz G. et al. 1371 (UJAT); F. Maldonado M. \& M.A. } \\
\text { de la Cruz A. } 45 \text { (UJAT); M.G. Dehesa J. } 35 \text { (UJAT); M.Á. Guadarrama } \\
\text { O. \& G. Ortiz G. } 854 \text { (UJAT); P. Tenorio L. 19405 et al. (UJAT); M.Á. } \\
\text { Guadarrama O. et al. 6272 (UJAT); C.P. Cowan \& M.Á. Guadarrama O. } \\
\text { 3927 (CSAT, MEXU); M.Á. Magaña A. } 1754 \text { (UJAT); M.Á. Guadarrama } \\
\text { O., G. Ortiz G. et al. 6816 (UJAT); M.Á. Guadarrama O., G. Ortiz G. et } \\
\text { al. 6822 (UJAT); M.Á. Guadarrama O., G. Ortiz G. et al. 5300 (UJAT); } \\
\text { M.Á. Guadarrama O., G. Ortiz G. et al. 6810 (UJAT); V. Ramón J. } 4 \\
\text { (UJAT); L. Ruíz V. } 10 \text { (UJAT); M.Á. Guadarrama O. \& F. López. 771 } \\
\text { (UJAT); R. Pérez M. } 6 \text { (UJAT); F. Ventura A. 21180 (MEXU); M.Á. } \\
\text { Guadarrama O. et al. 843, (UJAT). E. Matuda S/N (MEXU). }\end{array}$ & $\mathbf{L}$ \\
\hline $\begin{array}{l}\text { B. potosina (K.Schum. \& Loes.) } \\
\text { L.G. Lohmann }\end{array}$ & $\begin{array}{l}\text { Ibarra } \text { et al. } \text { (2015), Villaseñor ( 2016): } \\
\text { B. potosina }\end{array}$ & E. Matuda S/N (MEXU); A.M. Hanan A. 457 (MEXU, UJAT). & $\mathbf{L}$ \\
\hline Crescentia alata Kunth & $\begin{array}{l}\text { Pérez et al. (2005), Villaseñor (2016): } \\
\text { C. alata }\end{array}$ & C.M. Burelo R., M.Á. Guadarrama O., \& G.V. Ruiz L. 25 (UJAT). & $\mathbf{A}$ \\
\hline C. cujete $\mathrm{L}$. & $\begin{array}{l}\text { Cowan (1983), Pérez et al. (2005), } \\
\text { Villaseñor (2016): C. cujete }\end{array}$ & $\begin{array}{l}\text { C.M. Burelo R. } 28 \text { (UJAT); C.M. Burelo R. } 29 \text { (UJAT); A. Ramírez B. } \\
264 \text { (UJAT); M.Á. Guadarrama O. } 860 \text { (UJAT); A. Orozco } 430 \text { (CSAT); }\end{array}$ & $\mathbf{A}$ \\
\hline
\end{tabular}




\begin{tabular}{|c|c|c|c|}
\hline Especies & $\begin{array}{c}\text { Taxa incluidas en otros listados como } \\
\text { sinónimos o nombre en uso de } \\
\text { material de Tabasco } \\
\end{array}$ & Ejemplares examinados & $\mathbf{F V}$ \\
\hline & & $\begin{array}{l}\text { G. Ortiz G. } 5132 \text { (UJAT); R. Escolástico 147 (CSAT); M.A. Magaña A. } \\
1898 \text { (UJAT); R. González G. } 137 \text { (CSAT); H. Puig } 807 \text { (CSAT); C.P. } \\
\text { Cowan } 1786 \text { (CSAT); A.L. González C. (CSAT); M.Á. Guadarrama O. } \\
\& \text { G. Ortiz G. } 5193 \text { (UJAT); C.P. Cowan } 1880 \text { (CSAT); C.M. Burelo R., } \\
\text { M.Á. Guadarrama O. et al. } 47 \text { (UJAT). }\end{array}$ & \\
\hline $\begin{array}{l}\text { Cuspidaria inaequalis (DC. ex } \\
\text { Splitg.) L.G. Lohmann }\end{array}$ & $\begin{array}{l}\text { Cowan (1983), Pérez et al. (2005): } \\
\text { Arrabidaea inaequalis (DC. ex Splitg.) } \\
\text { Baill. } \\
\text { Villaseñor (2016): C. inaequalis }\end{array}$ & $\begin{array}{l}\text { F. Menéndez } 259 \text { (XAL); A. Novelo R. } 125 \text { (XAL); E. Matuda } 3421 \\
\text { (MICH). }\end{array}$ & $\mathbf{L}$ \\
\hline $\begin{array}{l}\text { Dolichandra quadrivalvis (Jacq.) } \\
\text { L.G. Lohmann }\end{array}$ & $\begin{array}{l}\text { Villaseñor (2016): Dolichandra } \\
\text { quadrivalvis }\end{array}$ & Ejemplar sin datos de colector (UJAT). & $\mathbf{L}$ \\
\hline D. uncata (Andrews) L.G. Lohmann & $\begin{array}{l}\text { Cowan (1983), Pérez et al. (2005): } \\
\text { Macfadyena uncata (Andrews) Sprague } \\
\text { \& Sandwith } \\
\text { Pérez et al. (2005), Villaseñor (2016): } \\
\text { D. uncata }\end{array}$ & $\begin{array}{l}\text { M.Á. Guadarrama O. \& G. Ortiz G. } 805 \text { (UJAT); G. Ortiz G. \& TCI-1 } \\
5142 \text { (UJAT); M.A. Magaña A. } 1883 \text { (UJAT); F. Ventura A. } 20454 \\
\text { (MEXU, UJAT); A.M. Hanan A. } 992 \text { (UJAT); F. David B. } 2813 D \text {, } \\
\text { (MEXU); C.M. Burelo R., M.Á. Guadarrama O. et al. } 45 \text { (UJAT). }\end{array}$ & $\mathbf{L}$ \\
\hline D. unguis-cati (L.) L.G. Lohmann & $\begin{array}{l}\text { Pérez } \text { et al. (2005), Villaseñor (2016): } \\
\text { D. unguis-cati }\end{array}$ & $\begin{array}{l}\text { F. Ventura A } 20169 \text { (MEXU, CSAT); R. Curiel } 4 \text { (CSAT); M.A. Magaña } \\
\text { A. \& S. Zamudio R. } 748 \text { (CSAT). }\end{array}$ & $\mathbf{L}$ \\
\hline $\begin{array}{l}\text { Fridericia candicans (Rich.) L.G. } \\
\text { Lohmann }\end{array}$ & Villaseñor (2016): Fridericia candicans & C. P. Cowan y M.A. Magaña A. 3221 (CSAT). & \\
\hline F. florida (DC.) L.G. Lohmann & Villaseñor (2016): $F$. florida & E. Hernández y C.L. Gilly $373(M E X U, M O)$ & \\
\hline $\begin{array}{l}\text { F. patellifera (Schltdl.) L.G. } \\
\text { Lohmann }\end{array}$ & $\begin{array}{l}\text { Cowan (1983), Pérez et al. } \mathbf{( 2 0 0 5 ) :} \\
\text { Petastoma patelliferum (Schltdl.) Miers } \\
\text { Ibarra } \text { et al. }(\mathbf{2 0 1 5}), \text { Villaseñor (2016): } \\
\text { F. patellifera }\end{array}$ & $\begin{array}{l}\text { M. Juárez P. } 200 \text { (UJAT) C.P. Cowan 1782 (CSAT); M.A. Magaña A. } \\
460 \text { (CSAT); M.A. Magaña A. et al. 1183, (CSAT). }\end{array}$ & $\mathbf{L}$ \\
\hline F. pubescens (L.) L.G. Lohmann & 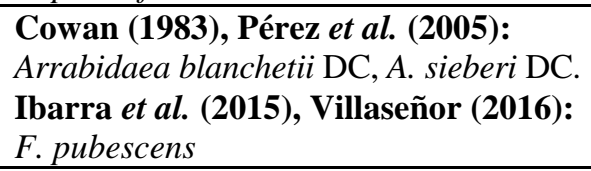 & M.A. Magaña A. 3221 (CSAT). & $\mathbf{L}$ \\
\hline $\begin{array}{l}\text { F. schumanniana (Loes.) L.G. } \\
\text { Lohmann }\end{array}$ & $\begin{array}{l}\text { Cowan (1983), Pérez et al. (2005): } \\
\text { Arrabidaea verrucosa (Standl.) } \\
\text { A.H.Gentry } \\
\text { Ibarra et al. (2015), Villaseñor (2016): } \\
\text { F. schumanniana }\end{array}$ & S. Zamudio R. \& M.A. Magaña A. 81 (CSAT); H. Puig 707 (CSAT). & $\mathbf{L}$ \\
\hline $\begin{array}{l}\text { Godmania aesculifolia (Kunth) } \\
\text { Standl. }\end{array}$ & Villaseñor (2016): G. aesculifolia & $\begin{array}{l}\text { M. Montoya G. } 311 \text { (UJAT); O. Téllez \& E. Martínez } 947 \text { (MEXU); M.A. } \\
\text { Magaña A. } 1004 \text { (CSAT). }\end{array}$ & $\mathbf{A}$ \\
\hline
\end{tabular}




\begin{tabular}{|c|c|c|c|}
\hline Especies & $\begin{array}{c}\text { Taxa incluidas en otros listados como } \\
\text { sinónimos o nombre en uso de } \\
\text { material de Tabasco } \\
\end{array}$ & Ejemplares examinados & $\mathbf{F V}$ \\
\hline $\begin{array}{l}\text { Handroanthus chrysanthus (Jacq.) } \\
\text { S.O. Grose }\end{array}$ & $\begin{array}{l}\text { Cowan (1983), Pérez et al. } \mathbf{( 2 0 0 5 ) :} \\
\text { Tabebuia chrysantha (Jacq.) } \\
\text { G.Nicholson } \\
\text { Villaseñor (2016): } \text { H. chrysanthus }\end{array}$ & H. Puig 782 (CSAT). & $\mathbf{A}$ \\
\hline H. guayacan (Seem.) S.O. Grose & $\begin{array}{l}\text { Cowan (1983), Pérez et al. (2005): } \\
\text { Tabebuia guayacan (Seem.) Hemsl. } \\
\text { Villaseñor (2016): H. guayacan }\end{array}$ & $\begin{array}{l}\text { C.M. Burelo R. } 30 \text { (UJAT); C.M. Burelo R. } 27 \text { (UJAT); P. Cazares C. } \\
301 \text { (UJAT); G. Ortiz G. } 40 \text { (UJAT); M.A. Magaña A. } 1522 \text { (UJAT); } \\
\text { C.P. Cowan et al. } 4657 \text { (CSAT); I. Madrigal D. } 28 \text { (UJAT). }\end{array}$ & $\bar{A}$ \\
\hline *Jacaranda mimosifolia D.Don & & $\begin{array}{l}\text { J.C. Jiménez S/N, (UJAT); E.S. López. H. \& S. Capello G. } 365 \text { (UJAT); } \\
\text { M.A. Magaña A. } 1512 \text { (MEXU, UJAT). }\end{array}$ & $\mathbf{A}$ \\
\hline $\begin{array}{l}\text { Mansoa sagotii (Bureau \& K. } \\
\text { Schum.) M.M. Silva-Castro. }\end{array}$ & $\begin{array}{l}\text { Cowan (1983): Adenocalymna } \\
\text { alboviolaceum Loes } \\
\text { Pérez et al. (2005): Mansoa hymenea, } \\
\text { A. alboviolaceum } \\
\text { Villaseñor (2016): Mansoa hymenea } \\
\text { (DC.) A.H.Gentry }\end{array}$ & $\begin{array}{l}\text { C. M. Burelo R., 54. (UJAT); C. M. Burelo R. } 55 \text { (UJAT, MEXU), C. M. } \\
\text { Burelo R. } 57 \text { (MEXU); S. Zamudio R., 140. (UJAT, MEXU). }\end{array}$ & $\mathbf{L}$ \\
\hline $\begin{array}{l}\text { M. verrucifera (Schlecht.) A.H. } \\
\text { Gentry }\end{array}$ & $\begin{array}{l}\text { Cowan (1983), Pérez et al. (2005), } \\
\text { Ibarra } \text { et al. (2015), Villaseñor (2016): } \\
\text { M. verrucifera }\end{array}$ & T. Croat 40122, (MO); F. Ventura, 20172 (MO, MEXU). & $\mathbf{L}$ \\
\hline $\begin{array}{l}\text { Martinella obovata (Kunth) Bureau } \\
\text { \& K.Schum. }\end{array}$ & $\begin{array}{l}\text { Cowan (1983), Pérez et al. } \mathbf{( 2 0 0 5 ) ,} \\
\text { Ibarra (2015), Villaseñor (2016): } M \text {. } \\
\text { obovata }\end{array}$ & $\begin{array}{l}\text { S. Zamudio R. } 39 \text { (MEXU, UJAT, CSAT); M.Á. Guadarrama O., G. } \\
\text { Ortiz G. \& S. Méndez, } 959 \text { (UJAT). }\end{array}$ & $\mathbf{L}$ \\
\hline $\begin{array}{l}\text { Parmentiera aculeata (Kunth) } \\
\text { Seem. }\end{array}$ & $\begin{array}{l}\text { Cowan (1983), Pérez et al. (2005), } \\
\text { Villaseñor (2016): P. aculeata }\end{array}$ & $\begin{array}{l}\text { C.M. Burelo R. } 35.5 \text { (UJAT); C.M. Burelo R. } 38 \text { (UJAT); S. Zamudio } \\
\text { R.124 (CSAT, MEXU); C. Romellón } 427 \text { (CSAT); M.A. Ramírez A. 610, } \\
\text { (CSAT); C.M. Burelo R. } 23 \text { (UJAT); D. Cruz C. } 30 \text { (UJAT); E. Chávez } \\
\text { 49, (UJAT); P. Alegría O. O6 (MEXU, UJAT); R. Curiel \& M.A } \\
\text { Guadarrama O. 107, (MEXU, CSAT); C.P. Cowan 1801 (CSAT); D. } \\
\text { Ricárdez C. } 138 \text { (CSAT); J.F. González S. } 46 \text { (UJAT); C.M. Burelo R. } 31 \\
\text { (UJAT); C.M. Burelo R. 50 (UJAT); M.Á. Guadarrama O., O. Castillo } \\
\text { A. \& A. Zubieta } 838 \text { (UJAT); L. González L. \& L. Hernández P. S/N } \\
\text { (MEXU). }\end{array}$ & $\mathbf{A}$ \\
\hline P. parviflora Lundell & $\begin{array}{l}\text { Cowan (1983), Pérez et al. }(\mathbf{2 0 0 5}) \text {, } \\
\text { Villaseñor (2016): } \text { P. parviflora } \\
\end{array}$ & $\begin{array}{l}\text { C.M. Burelo R. } 307 \text { (UJAT). E. Matuda } 3210 \text { (A, F, MICH); J.N. } \\
\text { Rovirosa } 171 \text { (K, US). }\end{array}$ & Ar \\
\hline $\begin{array}{l}\text { *Pyrostegia venusta (Ker Gawl.) } \\
\text { Miers. }\end{array}$ & & C.M. Burelo R. 56 (UJAT). & $\mathbf{A}$ \\
\hline $\begin{array}{l}\text { Roseodendron donnell-smithii } \\
\text { (Rose) Miranda }\end{array}$ & Villaseñor (2016): R. donnell-smithii & P. Cazares C. 293 (UJAT). & $\mathbf{A}$ \\
\hline
\end{tabular}




\begin{tabular}{|c|c|c|c|}
\hline Especies & $\begin{array}{l}\text { Taxa incluidas en otros listados como } \\
\text { sinónimos o nombre en uso de } \\
\text { material de Tabasco }\end{array}$ & Ejemplares examinados & $\mathbf{F V}$ \\
\hline *Spathodea campanulata P. Beauv. & Pérez et al. (2005): S. campanulata & $\begin{array}{l}\text { S. Carrillo P. } 15 \text { (CSAT); M.A. Magaña A. \& S. Zamudio R. } 159 \\
\text { (CSAT); A. López T. } 42 \text { (CSAT); J. Flores R. } 55 \text { (UJAT); C.M. Burelo R. } \\
25 \text { (UJAT); C.M. Burelo R. 26 (UJAT). }\end{array}$ & $\mathbf{A}$ \\
\hline $\begin{array}{l}\text { Stizophyllum riparium (Kunth) } \\
\text { Sandwith }\end{array}$ & $\begin{array}{l}\text { Ibarra } \text { et al. } \text { (2015), Villaseñor (2016): } \\
\text { S. riparium }\end{array}$ & J.L. Calzada 2327 (MEXU). & $\mathbf{L}$ \\
\hline $\begin{array}{l}\text { Tabebuia rosea (Bertol.) Bertero ex } \\
\text { A.DC. }\end{array}$ & $\begin{array}{l}\text { Cowan (1983): } T . \text { rosea, } T \text {. pentaphylla } \\
\text { (L.) Helms. } \\
\text { Pérez et al. (2005): Tabebuia rosea, } \\
\text { T. pentaphylla } \\
\text { Villaseñor (2016): } T \text {. rosea }\end{array}$ & $\begin{array}{l}\text { G. Ortiz G. O3 (UJAT); P. Cazares C. } 242 \text { (UJAT); M. H. Esmitia } 33 \\
\text { (CSAT); G. Ortiz G. 651 (UJAT); E. Romero S. } 19 \text { (UJAT); A. Orozco S. } \\
434 \text { (CSAT); M.Á. Guadarrama O., C.M. Burelo R. et al. } 6350 \text { (UJAT); } \\
\text { C.M. Burelo R. \& B. Pedraza V. } 32 \text { (UJAT); C.M. Burelo R. } 21 \text { (UJAT); } \\
\text { M.A. Magaña A. } 724 \text { (CSAT); S. Zamudio R. } 1393 \text { (UJAT); D. Ricardez, } \\
\text { C. } 183 \text { (MEXU, CSAT); C.P. Cowan } 1993 \text { (CSAT); P. Gómez R. } 04 \\
\text { (UJAT); N. Poot N. } 227 \text { (CSAT); N. Poot N. \& M.Á. Guadarrama O. } 309 \\
\text { (CSAT); G. Ramos E. \& M.Á. Guadarrama O., } 633 \text { (UJAT). }\end{array}$ & $\mathbf{A}$ \\
\hline $\begin{array}{l}\text { Tanaecium pyramidatum (Rich.) } \\
\text { L.G. Lohmann }\end{array}$ & $\begin{array}{l}\text { Pérez et al. (2005): Paragonia } \\
\text { pyramidata (Rich.) Bureau, Kongel } \\
\text { Villaseñor (2016): } \text { T. pyramidatum }\end{array}$ & $\begin{array}{l}\text { L. Calzada et al. } 2651 \text { (MEXU); G. Ortiz G. et al. } 5254 \text { (UJAT); G. Ortiz, } \\
\text { G. } 5284 \text { (UJAT); C.M. Burelo R. } 53 \text { (UJAT); A.M. Hanan A. } 514 \text { (UJAT, } \\
\text { MEXU). }\end{array}$ & $\mathbf{L}$ \\
\hline $\begin{array}{l}\text { Tanaecium tetragonolobum (Jacq.) } \\
\text { L.G.Lohmann }\end{array}$ & 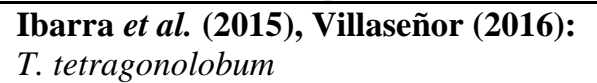 & M.Á. Guadarrama O. \& G. Ortiz G. 850 (MEXU, UJAT). & $\mathbf{L}$ \\
\hline *Tecoma capensis (Thunb.) Lindl. & $\begin{array}{l}\text { Cowan (1983), Pérez et al. (2005): } \\
\text { Tecoma capensis }\end{array}$ & C.M. Burelo R. 54 (UJAT). & Ar \\
\hline T. stans (L.) Juss. ex Kunth & $\begin{array}{l}\text { Cowan (1983), Pérez et al. (2005): } T \text {. } \\
\text { stans }\end{array}$ & $\begin{array}{l}\text { C.M. Burelo R. } 20 \text { (UJAT); C.M. Burelo R. } 35 \text { (UJAT); G. Ortiz G. } 37 \\
\text { (UJAT); C.M. Romellón A. S/N (UJAT); C. Romero } 175 \text { (CSAT); M.A. } \\
\text { Magaña A. \& C.P. Cowan } 1935 \text { (CSAT); H.C.Z. } 35 \text { (UJAT); A.B. } \\
\text { Sánchez V. } 35 \text { (CSAT); C.M. Burelo R. } 22 \text { (UJAT); Sin dato del colector } \\
\text { (UJAT); P. Alegría O. O7 (UJAT); R. Escolástico } 182 \text { (CSAT); R.L. } \\
\text { Ventura C. } 47 \text { (UJAT); M.Á. Guadarrama O., G. Ortiz G. et al. } 6848 \\
\text { (UJAT); S. Zamudio R. } 1294 \text { (CSAT); C.M. Burelo R. } 44 \text { (UJAT). }\end{array}$ & Ar \\
\hline Tynanthus guatemalensis Donn.Sm. & 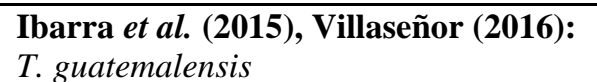 & $\begin{array}{l}\text { E. Matuda S/N (MEXU); F. Ventura A. } 21496 \text { (MEXU); A. Orozco, S. } \\
\text { Zamudio R., C.P. Cowan et al. } 2208 \text { (CSAT, MEXU). }\end{array}$ & $\mathbf{L}$ \\
\hline $\begin{array}{l}\text { Xylophragma seemannianum } \\
\text { (Kuntze) Sandwith }\end{array}$ & $\begin{array}{l}\text { Ibarra } \text { et al. }(\mathbf{2 0 1 5}), \text { Villaseñor (2016): } \\
\text { X. seemannianum }\end{array}$ & C.M. Burelo R. 36 (UJAT). & $\mathbf{L}$ \\
\hline
\end{tabular}

